Lorenz Peters, Christel Depienne*, and Stephan Klebe*

\title{
Familial adult myoclonic epilepsy (FAME): clinical features, molecular characteristics, pathophysiological aspects and diagnostic work-up
}

https://doi.org/10.1515/medgen-2021-2100

Received May 18, 2021; accepted November 16, 2021

\begin{abstract}
Familial adult myoclonic epilepsy (FAME) is a rare autosomal dominant disorder characterized by myoclonus and seizures. The genetic variant underlying FAME is an intronic repeat expansion composed of two different pentamers: an expanded TTTTA, which is the motif originally present at the locus, and an insertion of TTTCA repeats, which is usually located at the $3^{\prime}$ end and likely corresponds to the pathogenic part of the expansion. This repeat expansion has been identified so far in six genes located on different chromosomes, which remarkably encode proteins with distinct cellular localizations and functions. Although the exact pathophysiological mechanisms remain to be clarified, it is likely that FAME repeat expansions lead to disease independently of the gene where they occur. We herein review the clinical and molecular characteristics of this singular genetic disorder, which interestingly shares clinical features with other more common neurological disorders whose etiology remains mainly unsolved.
\end{abstract}

Keywords: familial adult myoclonic epilepsy, seizures, myoclonus, cortical tremor, repeat expansion, repeat insertion, intronic, AT-rich, long-read, molecular combing, fiber FISH

\section{Introduction}

Familial adult myoclonic epilepsy (FAME) is an autosomal dominant disorder characterized by a cortical myoclonic tremor and generalized seizures. It was first described in 1990 in Japan [1] but has meanwhile shown a worldwide distribution [2]. Over the past decades many alternative names like benign adult familial myoclonic epilepsy (BAFME), familial cortical myoclonic tremor with epilepsy (FCMTE), familial cortical myoclonic tremor (FCMT), famil-

\footnotetext{
*Corresponding authors: Christel Depienne, Institute of Human Genetics, University Hospital Essen, University of Duisburg-Essen, Essen, Germany, e-mail: christel.depienne@uni-due.de; and Stephan Klebe, Department of Neurology, University Hospital Essen, Essen, Germany, e-mail: stephan.klebe@uk-essen.de

Lorenz Peters, Department of Neurology, University Hospital Essen, Essen, Germany
}

ial essential myoclonus and epilepsy (FEME), familial benign myoclonus epilepsy of adult onset (FMEA) and heredofamilial tremor and epilepsy (HTE) have been proposed, reflecting its clinical heterogeneity. The latter underlines the difficulty to clearly distinguish this clinical entity from more frequently occurring, clinically overlapping disorders $[3,4]$. The exact prevalence of FAME remains largely unknown as this condition is probably often underdiagnosed, but it is estimated to be a rare disorder affecting less than one out of 35,000 individuals [5].

In the 20 years following its clinical description, several genetic loci have been mapped on different chromosomes, suggesting that FAME is genetically heterogeneous. However, recent evidence suggests that the cause of FAME is actually linked to the same pathogenic repeat expansion in different genes and that the pathophysiological mechanisms are likely independent from the gene where the expansion occurs or its functions. In this setting, FAME is becoming a paradigm illustrating how pathogenic variants may lead to the same disease irrespectively of their location in the genome.

In the present review, we give an overview of the genetics, clinical features, genotype-phenotype correlations and diagnostic work-up of FAME.

\section{Clinical presentation: predominant cortical myoclonus and epilepsy}

FAME symptoms usually occur in the second or third decade with a highly variable age of onset (range, 3-70 years) [2]. Epidemiologic data about the penetrance are not available but approximately 150 families with FAME are reported so far [6]. It is assumed that there are many more FAME families that remain undiagnosed due to mild and/or overlooked symptoms or misdiagnosis with more frequent disorders. The disease course of FAME is usually slowly progressive with a disease duration over several decades $[7,8]$.

\section{Cortical myoclonus}

The defining symptom and most prominent movement disorder of FAME is cortical myoclonus. Myoclonus itself is 
Table 1: Comparison between symptoms, EMG and therapy in essential tremor and cortical myoclonus.

\begin{tabular}{|c|c|c|c|c|c|}
\hline Syndrome & Localization & Frequency & EMG pattern & Therapy & Other features \\
\hline Essential tremor & $\begin{array}{l}\text { UE with or without other } \\
\text { locations (voice; LE), } \\
\text { predominantly bilateral }\end{array}$ & $4-12 \mathrm{~Hz}$ & $\begin{array}{l}\text { rhythmic; commonly } \\
\text { synchronized }\end{array}$ & $\begin{array}{l}\text { Propranolol, Primidone, } \\
\text { Topiramate }\end{array}$ & $\begin{array}{l}\text { No further neurological } \\
\text { signs (if further } \\
\text { neurological signs occur } \\
\text { [e.g., ataxia] the } \\
\text { classification is } \\
\text { "essential tremor plus") }\end{array}$ \\
\hline Cortical myoclonus & $\begin{array}{l}\text { UE (unilateral or } \\
\text { bilateral) }\end{array}$ & $9-18 \mathrm{~Hz}$ & $\begin{array}{l}\text { arrhythmic bursts of } \\
\text { around } 15 \text { to } 60 \mathrm{~ms}\end{array}$ & $\begin{array}{l}\text { AED (e.g., } \\
\text { Levetiracetam, sodium } \\
\text { valproate) }\end{array}$ & $\begin{array}{l}\text { Cortical excitability } \\
\text { (giant SEP, jerk locked } \\
\text { EEG changes) }\end{array}$ \\
\hline
\end{tabular}

UE, upper extremities; LE, lower extremities; AED, antiepileptic drugs; Hz, hertz; SSEP, somatosensory-evoked potentials; EEG, electroencephalography

defined as a "complex hyperkinetic movement disorder characterized by sudden, brief, involuntary jerks of a single muscle or a group of muscles" [9]. The origin of myoclonic jerks can be peripheral, spinal, subcortical or cortical as in FAME patients [9]. The cortical myoclonus predominantly affects the distal upper limbs in posture or action, with a variable severity between and within pedigrees. However, it may also affect other body parts like lower extremities, head, trunk, proximal upper limbs and facial muscles (e. g., eyelids). Myoclonus is often provoked by a startle reaction and worsened by goal-directed movements. Markedly, the movements are occasionally interrupted by bigger myoclonic jerks predominantly in the upper limbs.

Clinically, due to its shivering-like small jerks and high frequency (approx. 10 per second) the irregularity is not easy to recognize; therefore, it is sometimes misdiagnosed as essential tremor (ET) (Table 1, Fig. 2) or enhanced physiological tremor. ET represents the most frequent movement disorder in neurology with a presumed prevalence of $0.4 \%$ to $6.3 \%$ worldwide [10] and frequently shows a positive family history [11]. In contrast to FAME, it has not yet been possible to find one or several genes linked to ET despite positive results in linkage analyses [12]. For clinicians it is therefore important to distinguish between FAME, other types of myoclonic jerks and ET. To ensure differentiation between ET and FAME, the term "tremor" should be replaced by rhythmic cortical myoclonus in FAME patients.

\section{Epilepsy}

Seizure onset age is variable, ranging between 12 and 67 years, with a mean age at onset of 30 years [13]. In FAME1 and FAME2, seizures usually start a few years after myoclonus onset, whereas in patients with expansions in the MARCHF6 gene (FAME3), seizures may occur before or at the same time as cortical myoclonus in some family members [7]. In some FAME pedigrees, anticipation has been observed, at least for the age at epilepsy onset $[7,14,15]$. As in other primary generalized epilepsies (GE), seizures in FAME patients are commonly exacerbated or triggered by photic stimulation, alcohol or sleep deprivation [16]. Seizures tend to be less frequent compared to other forms of GE, but there are reports of intractable courses, which require a poly-antiepileptic drug regimen [5]. Absences are rare but were described in an Italian pedigree [17]. Epilepsies with a FAME etiology must be distinguished from juvenile myoclonic epilepsy (JME) and progressive myoclonus epilepsy when tremor is not a key distractor. In JME, the myoclonic-like seizures often occur in teens or young adults during wakeup in the morning. The deleterious progression and the occurrence of ataxia and dementia is a hallmark of progressive myoclonus epilepsy and will help to differentiate from FAME during the further time course. More rarely, other symptoms may be present in FAME patients including cognitive decline or intellectual disability, migraine and night blindness $[4,13]$.

\section{Electrophysiology and brain imaging}

Cortical myoclonus is generated by abnormal neuronal discharges in cortical layers. The discharges lead to a brief activation of the corticospinal tract. In contrast, negative myoclonus is characterized by a sudden pause of corticospinal tract activity. A negative myoclonus is also known as asterixis or "flapping tremor." 
Table 2: List of genes containing pathogenic TTTTA/TTTCA repeat expansions related to FAME.

\begin{tabular}{|c|c|c|c|c|c|c|}
\hline Chr. & Gene name & Intron & Genomic coordinates (hg38) & Normal alleles repeat range & Pathogenic alleles repeat range & Ref. \\
\hline 2 & STARD7 & 1 & $96197067-96197121$ & TTTA(TTTTA $)_{9-20}$ TTTT & $(\text { TTTTA/TTTCA })_{661-735}$ & {$[14]$} \\
\hline 3 & YEATS2 & 1 & $183712192-183712226$ & TTTTATGTTC(TTTTA $)_{7}$ TTTTTT & (TTTTA/TTTCA) $\exp$ & [27] \\
\hline 4 & RAPGEF2 & $14 / 20^{*}$ & $159342529-159342618$ & $(\text { TTTTA })_{5}($ TATTA $)(\text { TTTTA })_{12-14}$ & (TTTTA/TTTCA $)_{\exp }$ & [23] \\
\hline 5 & MARCHF6 & 1 & $10356339-10356411$ & TTTTTTATTTA(TTTTA) ${ }_{10-30}$ TTTT & (TTTTA/TTTCA) $660-2,800$ & [7] \\
\hline 8 & SAMD12 & 4/3'UTR* & $118366813-118366915$ & $(\mathrm{TTTTA})_{7}(\mathrm{TTA})(\mathrm{TTTTA})_{13-\exp }$ & (TTTTA/TTTCA $)_{440-3,680}$ & [23] \\
\hline 16 & TNRC6A & 1 & $24703039-24703078$ & $(\text { TTTTA })_{8}$ & (TTTTA/TTTCA) $\exp$ & [23] \\
\hline
\end{tabular}

Chr.: chromosome; Ref.: reference; ${ }^{\star}$ : location varies depending on the isoform

The electrophysiology of cortical myoclonus is based on electromyographical measurements (EMG), somatosensory-evoked potentials (SSEP) and long latency stretch reflexes (LLSRs). In the EMG, the distinction is made based on the frequency spectrum and the duration of the EMG burst. In patients with a central tremor component (e.g., ET, Parkinson's disease) a synchronized specific frequency with a strong peak in the EMG power spectrum is observed. In contrast, the EMG of myoclonus reveals arrhythmic bursts of variable duration. The irregularity with short burst-like potentials will help to guide the clinician toward a myoclonus, which is often stimulussensitive. The burst duration in cortical myoclonus is shorter $(<100 \mathrm{~ms})$ compared to subcortical generated myoclonus.

Backaveraging of simultaneous EMG and EEG can detect that cortical discharges on EEG precede the jerks seen on EMG [18]. The SSEP may present giant cortical responses [19] as another sign of the cortical origin of the myoclonus. In addition, a reduction in the resting motor threshold intensity and the post-motor-evoked potential silent period has been reported in a few patients evaluated by transcranial magnetic stimulation, indicating that central motor inhibitory mechanisms are impaired in these cases [20]. Interestingly the cerebellum seems to play a role in the generation of cortical myoclonus via cerebellothalamo-cortical projections that could change the gain of sensorimotor connections [21]. Long loop responses called C-reflexes which are elicited at rest are often detectable in FAME patients, indicating an increased cortical excitability [13].

Brain imaging studies, especially MRI, regularly did not show any consistent abnormalities. Proton magnetic resonance spectroscopy has reported an elevated choline/creatine ratio in the cerebellum cortex of patients from an Italian pedigree linked to chromosome 2 (FAME2) compared with controls [22]. Interestingly, STARD7 encodes a member of the StAR-related lipid transfer (START) domain-containing family of lipid transfer proteins whose functions include intra-mitochondrial lipid transfer of phosphatidylcholine [14]. So far, it is not clear if increased choline/creatine ratios are restricted to FAME2 patients, but if so, this could indicate an alteration of the mitochondrial function of STARD7 in brains of FAME2 subjects.

\section{Current treatment}

Since there are only anecdotal data on pharmacological interventions in FAME available, treatment options rely on general guidelines for symptomatic treatment of cortical myoclonus [9], which overlaps with treatment for epilepsies. First line treatment should include levetiracetam, sodium valproate and/or benzodiazepines.

\section{Genetic basis: a dominant disorder resulting from the same noncoding repeat expansion in at least six different genes}

Four genetic loci for FAME have been identified by linkage analysis (2p11-2q11, 3q26-q28, 5p15, 8q24) in the past 20 years but the underlying genetic cause remained unknown despite extensive sequencing of genes in the candidate intervals. The decisive breakthrough was achieved when intronic repeat expansions in sterile alpha motif domain containing 12 (SAMD12) (FAME1) were identified as the main cause of BAFME/FAME in Japan [23]. This accomplishment was possible thanks to the reduction of the candidate interval on chromosome 8 to a single gene. Expansions composed of two different pentamer repeat motifs, TTTTA, which is the motif normally present at this locus, and TTTCA, which corresponds to an insertion, were identified based on non-Mendelian inheritance of the corresponding region due to the nonamplification of the ex- 
panded allele using standard PCR conditions [23]. SAMD12 expansions are associated with a specific haplotype originating from a common Asian ancestor and account for a large proportion of FAME families in China, India and Sri Lanka $[8,23,24,25,26]$. In two Japanese families without $S A M D 12$ expansions, similar expansions in two other genes, TNRC6A on chromosome 16 (FAME6) and RAPGEF2 on chromosome 4 (FAME7), were identified in a small family and a sporadic case, respectively [23]. A pathogenic expansion in RAPGEF2 was further identified in another FAME family of Chinese origin [24].

The identification of SAMD12 expansions in FAME1 was followed by similar discoveries at the three other loci: TTTTA/TTTCA expansions were identified in introns of STARD7 on chromosome 2 [14], MARCHF6 (formerly MARCH6) on chromosome 5 [7] and YEATS2 on chromosome 3 [27]. Altogether, similar intronic TTTTA/TTTCA repeat expansions have thus far been identified in six different genes (Fig. 1, Table 2). In all genes, expansions replace a polymorphic primate-specific microsatellite originally composed of TTTTA repeats, usually adjacent to one or more Alu elements (Fig. 1). The size of the expansions is variable, ranging from 2 to $18 \mathrm{~kb}$ with an average of $4-5 \mathrm{~kb}$ (i. e., 800-1,000 repeats). There exists an inverse correlation between the age at onset and the overall size of the expansion [7, 23], which is mainly determined by the length of the TTTCA repeats [7]. Expanded SAMD12 alleles exclusively composed of TTTTA repeats exist in a subset of healthy Japanese individuals, which further suggests that TTTCA repeats constitute the pathogenic part of the expansion while TTTTA repeats are nonpathogenic [23].

FAME repeat expansions are highly unstable in a length-dependent manner. The characterization of MARCHF6 expansions using molecular combing (fiber FISH) has revealed that individual expanded alleles vary considerably in length and structure, with expansion size ranging from 1.7 to $36 \mathrm{~kb}$ in blood cells of a same individual. Furthermore, expansions exceeding $10 \mathrm{~kb}$, present in individuals with the most severe phenotypes, were associated with microrearrangements at the site of the expansion, representing up to $10 \%$ of alleles with an expansion [7]. These results suggest that FAME expansions constitute fragile sites prone to chromosomal breakage.

Interestingly, a large Chinese FAME family carrying a SAMD12 expansion composed of a TTTGA insertion instead of the TTTCA repeats was reported, suggesting that other motifs than TTTCA could also be pathogenic [28]. However, a recent study has shown that TTTCA and TTTGA insertions coexist in some expansions [29], and therefore sequencing of the full expansion by a long-read technol- ogy taking the high degree of mosaicism into account is of utmost importance.

\section{Diagnostic work-up}

The clinical diagnosis of FAME is based on: (1) the patient's clinical history, which is usually suggestive of autosomal dominant inheritance; (2) the association of cortical myoclonus and seizures, either in the patient or within the family; and/or (3) a cortical origin of the tremor demonstrated by backaveraging of simultaneous EMG and EEG. However, this diagnosis should also be considered for patients without family history as sporadic cases have also been occasionally reported. ET and JME, which are the two main differential diagnoses, should be considered and ruled out based on seizure semiology or electrophysiological investigations (EEG, EMG and SEPs).

Identification of the molecular cause both allows to confirm the clinical diagnosis and enables genetic counseling in the family. Genetic testing usually requires a blood sample used to extract genomic DNA and perform repeat-primed PCR (RP-PCR) assays for the three main loci (FAME1, FAME2, FAME3). Alternative technologies, including molecular combing, nanopore sequencing and/or optical genome mapping, may also be used although these techniques are so far often limited to research studies. Repeat expansions linked to FAME can also be detected from genome data using specific bioinformatics tools such as ExpansionHunter [30]. However, a confirmation that the expansion includes pathogenic TTTCA repeats using either RP-PCR or a sequencing technique is recommended.

\section{Pathophysiological mechanisms leading to FAME}

The identification of similar pentameric expansions in introns of unrelated genes strongly suggests that the pathological mechanisms are independent of the gene or its function. This hypothesis is further supported by the completely different functions and expression profiles of the six genes where FAME expansions occur: SAMD12 is a gene of unknown function encoding three differently spliced isoforms, the largest one being predominantly expressed in the cortex and cerebellum [23]. Rap guanine nucleotide exchange factor 2 (RAPGEF2) encodes several isoforms primarily expressed in neurons, with highest expression in the cortex. These proteins belong to the RAS subfamily 


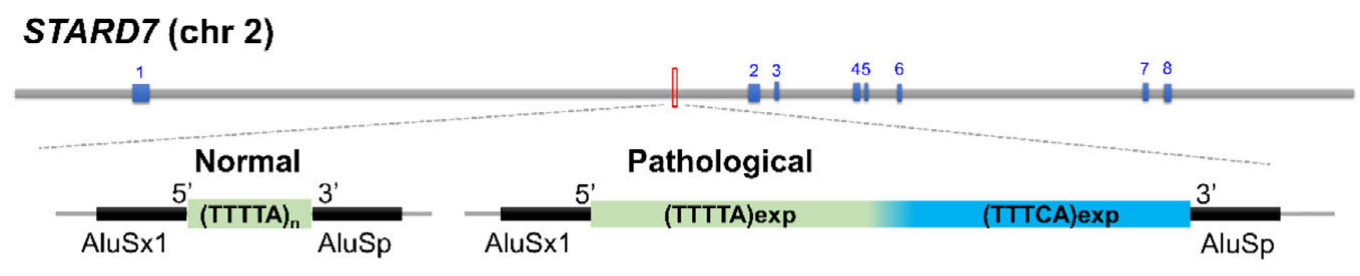

\section{YEATS2 (chr 3)}
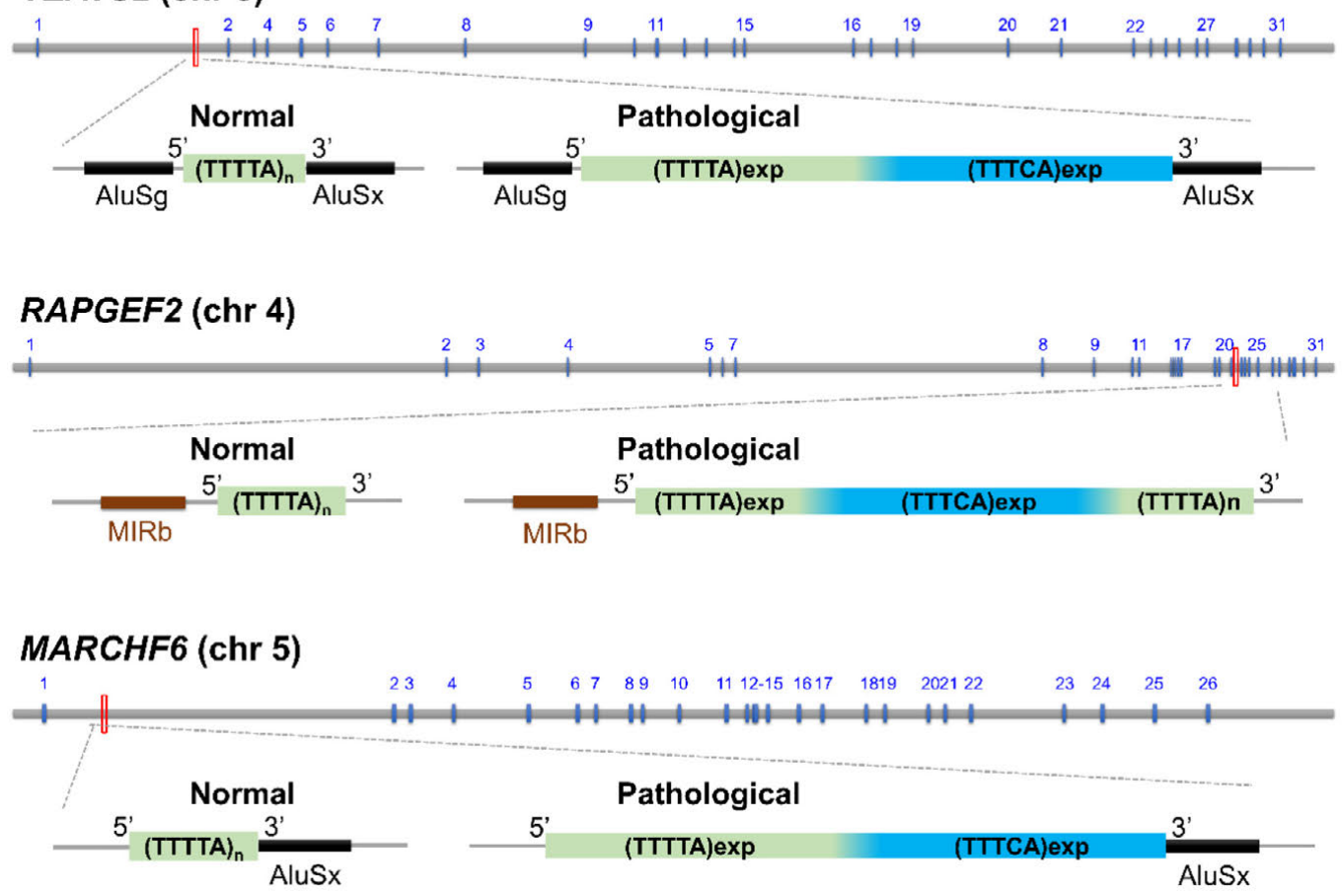

\section{SAMD12 (chr 8)}

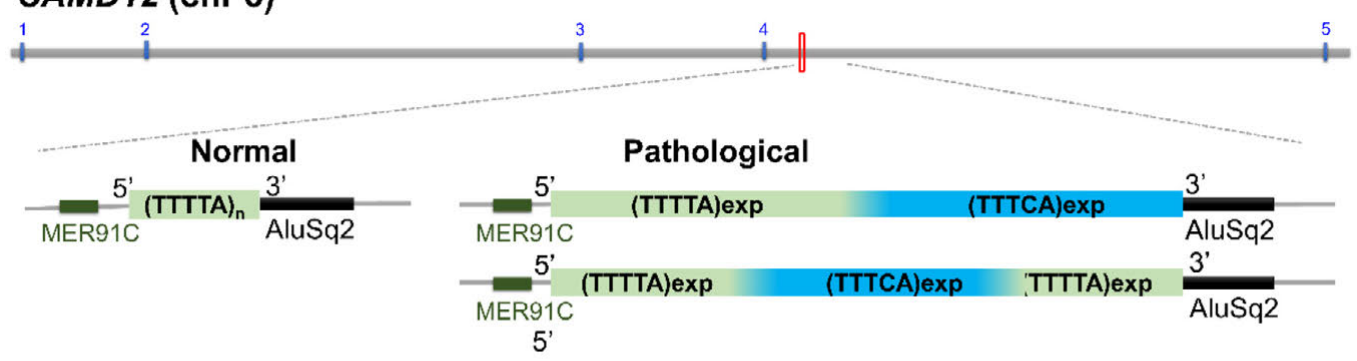

TNRC6A (chr 16)

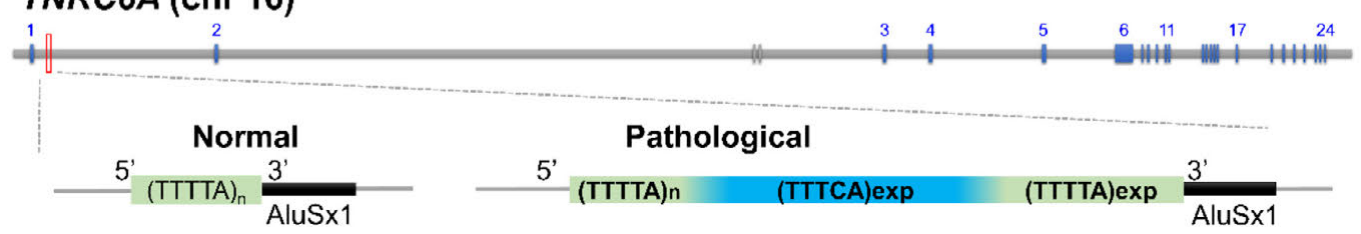

Figure 1: FAME is caused by similar pentameric repeat expansion in six different genes (STARD7, YEATS2, RAPGEF2, MARCHF6, SAMD12, TNRC6A) located on different chromosomes. These expansions always occur at a polymorphic short tandem repeat (microsatellite) site initially composed of TTTTA motifs (in green) in healthy individuals (on the left). This microsatellite is usually adjacent to one or more Alu elements. Pathogenic repeat expansions (on the right) always include a TTTCA repeat insertion (in blue) that likely corresponds to the diseaseassociated part of the expansions and is mainly located at the $3^{\prime}$ end of the expansion. Configurations with this insertion in a more central position have also been described in SAMD12. All expansions are intronic and are likely transcribed as part of pre-mRNA transcripts. They could lead to a toxic RNA able to accumulate and trap RNA-binding proteins, although this remains to be demonstrated. 

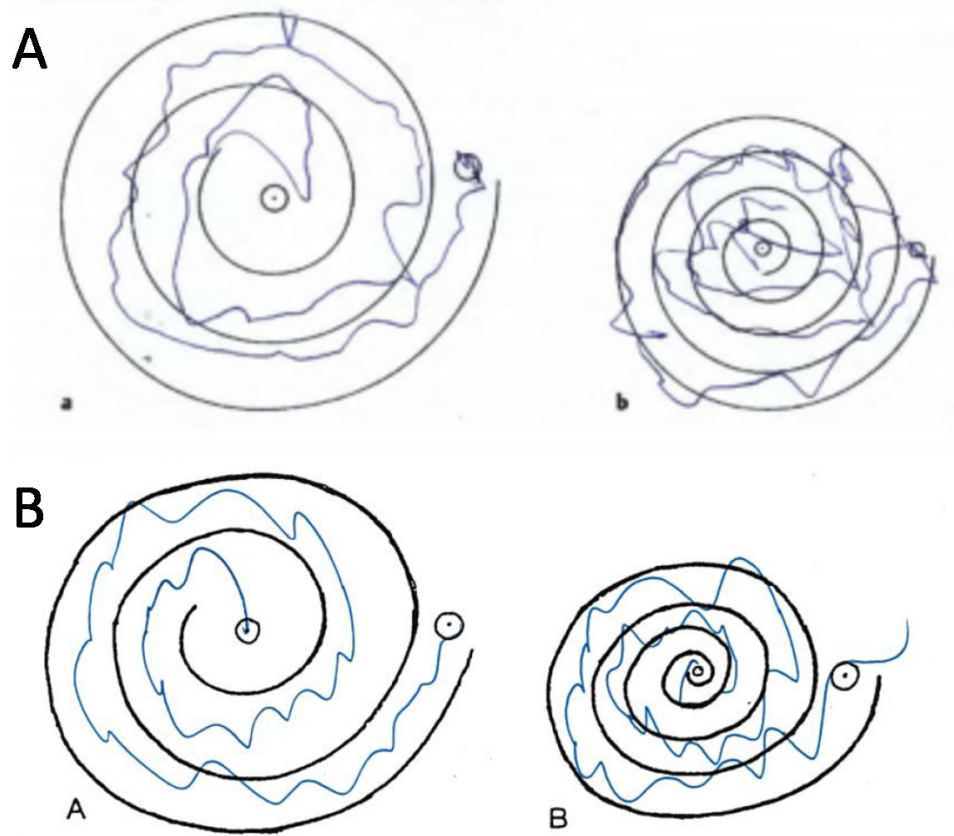

Figure 2: Archimedes spiral test of a FAME patient (A) (from Florian et al. [7]) and a moderately affected patient with ET (B). The figure shows the poor clinical differentiation between cortical myoclonus (FAME; A) and tremor (ET; B).

of GTPases and act to switch on Ras and/or ERK signaling pathways in response to the activation of cell surface receptors, such as dopaminergic receptors [31, 32]. Trinucleotide repeat-containing gene $6 \mathrm{~A}$ (TNRC6A), also called glycine/tryptophane repeat protein (GW182), encodes a component of a cytoplasmic ribonucleoprotein complex involved in the regulation of mRNA silencing, stability and translation that is ubiquitously expressed, with highest expression in the cerebellum $[33,34]$. MARCHF6 encodes a ubiquitously expressed E3 ubiquitin ligase that mediates the degradation of misfolded or damaged proteins in the endoplasmic reticulum [35, 36]. STARD7 encodes a ubiquitous protein involved in lipid transport and metabolism [37]. YEATS2 encodes a ubiquitous subunit of the ADA2Acontaining histone acetyltransferase complex, with high expression in the cerebellum [38]. Some genes are specifically expressed in the central nervous system, while others are ubiquitously expressed, but all six are expressed in the human brain, including the cortex and the cerebellum.

Since expansions are all intronic, expanded repeats should be transcribed as part of precursor mRNA (premRNA) transcripts. However, contradictory results have been obtained regarding the expression of UUUUA/UUUCA repeats. On the one hand, reads corresponding to SAMD12 transcripts filled with UUUUA/UUUCA repeats have been detected in association with RNA foci and abortive transcription in post-mortem brains of Japanese patients [23]. On the other hand, despite the ubiquitous expression of MARCHF6 and STARD7, no reads filled with UUUUA or UUUCA repeats could be detected in lymphoblasts or fibroblasts of patients with expansions in these genes [7, 14]. Furthermore, the expression and splicing of MARCHF6 and STARD7 were unaltered in these tissues compared to unaffected controls, and there was no increase in MARCHF6 intron 1 retention, which would be expected if abortive transcription and RNA accumulation would occur $[7,14]$. These discrepant results suggest that abnormal transcription and accumulation of UUUCA repeats observed in SAMD12 expansion carriers could be restricted to neuronal tissues or do not exist in other FAME subtypes, and additional studies are required to determine whether RNA foci exist in other FAME subtypes.

Remarkably, TTTTA/TTTCA expansions in intron 3 of $D A B 1$ have initially been associated with spinocerebellar ataxia 37 (SCA37) [39]. DAB1 encodes a downstream effector of the reelin signaling pathway, contributing to the correct positioning of neurons in the developing brain. Its expression is higher in the cerebellum than in the cortex, possibly explaining why expansions at this locus are specifically associated with cerebellar ataxia. However, several FAME-associated genes (e.g., TNRC6A, YEATS2) are also highly expressed in the cerebellum, suggesting that the expression of the genes where expansions occur is not the only factor influencing the phenotypic pre- 
sentation. A recent study based on post-mortem brains of SCA37 expansion carriers revealed that SCA37 expansions increase $D A B 1$ expression and trigger alternative splicing events favoring the inclusion of two exons absent from isoforms normally present in the brain [40]. Yet, SCA37 and FAME expansions appear to have different characteristics: FAME TTTCA repeats are predominantly located at the $5^{\prime}$ extremity of the expansion, whereas SCA37 TTTCA repeats are inserted in the middle of TTTTA repeats. SCA37 expansions also tend to be shorter in size (usually up to $1 \mathrm{~kb}$ ), while the shortest FAME expansion described so far was above $2 \mathrm{~kb}$. Interestingly, nonpathogenic expansions up to $3 \mathrm{~kb}$ composed of TTTTA repeats only also exist at the $D A B 1$ locus, further confirming that homogeneous TTTTA expansions are benign, independently of the locus.

\section{Conclusions}

Recent genetic evidence revealed that FAME is a repeat expansion disorder involving the same AT-rich pentameric motifs in distinct genes. The description of pedigrees without expansions in one of the six known loci argues for the existence of additional loci of this repeat expansion [7]. Further research studies are necessary to apprehend the mechanisms by which these expansions are pathogenic but the observation of RNA foci in FAME1 [23] argues for possible RNA-mediated toxicity that remains to be proven in other FAME subtypes. Due to its rarity and overlap with more frequently occurring neurological disorders, FAME patients are likely often misdiagnosed. Diagnostic consensus criteria for FAME remain to be redefined as, to our knowledge, the available criteria have been proposed in 2005 and 2006 [41, 42], i. e., long before the identification of its genetic cause.

Acknowledgments: The authors thank the two anonymous reviewers of this manuscript for their valuable comments.

Research funding: The research studies conducted by the authors are supported by University Hospital Essen, the Deutsche Forschungsgemeinschaft (DFG), Fondation Maladies Rares and the Tom-Wahlig Stiftung.

Author contributions: All authors have accepted responsibility for the entire content of this manuscript and approved its submission.

Competing interests: Authors state no conflict of interest. Informed consent: Informed consent was obtained from all individuals included in this study.
Ethical approval: The local Institutional Review Board deemed the study exempt from review.

\section{References}

[1] Ikeda A, Kakigi R, Funai N, Neshige R, Kuroda Y, Shibasaki $H$. Cortical tremor: a variant of cortical reflex myoclonus. Neurology. 1990;40(10):1561-5.

[2] van den Ende T, Sharifi S, van der Salm SMA, van Rootselaar AF. Familial cortical myoclonic tremor and epilepsy, an enigmatic disorder: from phenotypes to pathophysiology and genetics. A systematic review. Tremor Other Hyperkinet Mov (N Y). 2018;8:503.

[3] Cen ZD, Xie F, Xiao JF, Luo W. Rational search for genes in familial cortical myoclonic tremor with epilepsy, clues from recent advances. Seizure. 2016;34:83-9.

[4] van Rootselaar AF, van Schaik IN, van den Maagdenberg MA, Koelman JH, Callenbach PM, Tijssen MA. Familial cortical myoclonic tremor with epilepsy: a single syndromic classification for a group of pedigrees bearing common features. Mov Disord. 2005;20(6):665-73.

[5] Lagorio I, Zara F, Striano S, Striano P. Familial adult myoclonic epilepsy: a new expansion repeats disorder. Seizure. 2019;67:73-7.

[6] Ishiura H, Tsuji S. Advances in repeat expansion diseases and a new concept of repeat motif-phenotype correlation. Curr Opin Genet Dev. 2020;65:176-85.

[7] Florian RT, Kraft F, Leitao E, Kaya S, Klebe S, Magnin E et al. Unstable TTTTA/TTTCA expansions in MARCH6 are associated with familial adult myoclonic epilepsy type 3. Nat Commun. 2019;10(1):4919.

[8] Bennett MF, Oliver KL, Regan BM, Bellows ST, Schneider AL, Rafehi $\mathrm{H}$ et al. Familial adult myoclonic epilepsy type 1 SAMD12 TTTCA repeat expansion arose 17,000 years ago and is present in Sri Lankan and Indian families. Eur J Hum Genet. 2020;28(7):973-8.

[9] Zutt R, van Egmond ME, Elting JW, van Laar PJ, Brouwer OF, Sival DA et al. A novel diagnostic approach to patients with myoclonus. Nat Rev Neurol. 2015;11(12):687-97.

[10] Louis ED, Ferreira JJ. How common is the most common adult movement disorder? Update on the worldwide prevalence of essential tremor. Mov Disord. 2010;25(5):534-41.

[11] Louis ED. The essential tremors: evolving concepts of a family of diseases. Front Neurol. 2021;12:650601.

[12] Hopfner F, Helmich RC. The etiology of essential tremor: genes versus environment. Parkinsonism Relat Disord. 2018;46(Suppl 1):S92-6.

[13] Striano P, Zara F. Autosomal dominant cortical tremor, myoclonus and epilepsy. Epileptic Disord. 2016;18(S2):139-44.

[14] Corbett MA, Kroes T, Veneziano L, Bennett MF, Florian R, Schneider AL et al. Intronic ATTTC repeat expansions in STARD7 in familial adult myoclonic epilepsy linked to chromosome 2. Nat Commun. 2019;10(1):4920.

[15] Crompton DE, Sadleir LG, Bromhead CJ, Bahlo M, Bellows ST, Arsov $T$ et al. Familial adult myoclonic epilepsy: recognition of mild phenotypes and refinement of the $2 q$ locus. Arch Neurol. 2012;69(4):474-81. 
[16] Uyama E, Fu YH, Ptacek LJ. Familial adult myoclonic epilepsy (FAME). Adv Neurol. 2005;95:281-8.

[17] Elia M, Musumeci SA, Ferri R, Scuderi C, Del Gracco S, Bottitta $M$ et al. Familial cortical tremor, epilepsy, and mental retardation: a distinct clinical entity? Arch Neurol. 1998;55(12):1569-73.

[18] Shibasaki H, Yamashita Y, Kuroiwa Y. Electroencephalographic studies myoclonus. Brain. 1978;101(3):447-60.

[19] Rothwell JC, Obeso JA, Marsden CD. On the significance of giant somatosensory evoked potentials in cortical myoclonus. J Neurol Neurosurg Psychiatry. 1984;47(1):33-42.

[20] Guerrini R, Bonanni P, Patrignani A, Brown P, Parmeggiani L, Grosse $P$ et al. Autosomal dominant cortical myoclonus and epilepsy (ADCME) with complex partial and generalized seizures: a newly recognized epilepsy syndrome with linkage to chromosome 2p11.1-q12.2. Brain. 2001;124(Pt 12):2459-75.

[21] Ganos C, Kassavetis P, Erro R, Edwards MJ, Rothwell J, Bhatia $K P$. The role of the cerebellum in the pathogenesis of cortical myoclonus. Mov Disord. 2014;29(4):437-43.

[22] Striano P, Caranci F, Di Benedetto R, Tortora F, Zara F, Striano

S. (1)H-MR spectroscopy indicates prominent cerebellar dysfunction in benign adult familial myoclonic epilepsy. Epilepsia. 2009;50(6):1491-7.

[23] Ishiura H, Doi K, Mitsui J, Yoshimura J, Matsukawa MK, Fujiyama $A$ et al. Expansions of intronic TTTCA and TTTTA repeats in benign adult familial myoclonic epilepsy. Nat Genet. 2018;50(4):581-90.

[24] Lei XX, Liu Q, Lu Q, Huang Y, Zhou XQ, Sun HY et al. TTTCA repeat expansion causes familial cortical myoclonic tremor with epilepsy. Eur J Neurol. 2019;26(3):513-8.

[25] Cen Z, Jiang Z, Chen Y, Zheng X, Xie F, Yang X et al. Intronic pentanucleotide TTTCA repeat insertion in the SAMD12 gene causes familial cortical myoclonic tremor with epilepsy type 1 . Brain. 2018;141(8):2280-8.

[26] Zeng S, Zhang MY, Wang XJ, Hu ZM, Li JC, Li N et al. Long-read sequencing identified intronic repeat expansions in SAMD12 from Chinese pedigrees affected with familial cortical myoclonic tremor with epilepsy. J Med Genet. 2019;56(4):265-70.

[27] Yeetong P, Pongpanich M, Srichomthong C, Assawapitaksakul A, Shotelersuk V, Tantirukdham $N$ et al. TTTCA repeat insertions in an intron of YEATS2 in benign adult familial myoclonic epilepsy type 4. Brain. 2019;142(11):3360-6.

[28] Cen Z, Chen Y, Yang D, Zhu Q, Chen S, Chen X et al. Intronic (TTTGA)n insertion in SAMD12 also causes familial cortical myoclonic tremor with epilepsy. Mov Disord. 2019;34(10):1571-6.

[29] Mizuguchi T, Toyota T, Miyatake S, Mitsuhashi S, Doi H, Kudo $\mathrm{Y}$, et al. Complete sequencing of expanded SAMD12 repeats by long-read sequencing and Cas9-mediated enrichment. Brain. 2021.

[30] Dolzhenko E, van Vugt J, Shaw RJ, Blitterswijk MA, van Blitterswijk M, Narzisi G et al. Detection of long repeat expansions from PCR-free whole-genome sequence data. Genome Res. 2017;27(11):1895-903.

[31] Jiang SZ, Xu W, Emery AC, Gerfen CR, Eiden MV, Eiden LE. NCS-Rapgef2, the protein product of the neuronal Rapgef2 gene, is a specific activator of D1 dopamine receptor-dependent ERK phosphorylation in mouse brain. eNeuro. 2017;4(5)
[32] Emery AC, Eiden MV, Mustafa T, Eiden LE. Rapgef2 connects GPCR-mediated cAMP signals to ERK activation in neuronal and endocrine cells. Sci Signal. 2013;6(281):ra51.

[33] Schneider MD, Najand N, Chaker S, Pare JM, Haskins J, Hughes SC et al. Gawky is a component of cytoplasmic mRNA processing bodies required for early Drosophila development. J Cell Biol. 2006;174(3):349-58.

[34] Nishi K, Nishi A, Nagasawa T, Ui-Tei K. Human TNRC6A is an Argonaute-navigator protein for microRNA-mediated gene silencing in the nucleus. RNA. 2013;19(1):17-35.

[35] Zattas D, Berk JM, Kreft SG, Hochstrasser M. A conserved C-terminal element in the yeast Doa10 and human MARCH6 ubiquitin ligases required for selective substrate degradation. J Biol Chem. 2016;291(23):12105-18.

[36] Stefanovic-Barrett S, Dickson AS, Burr SP, Williamson JC, Lobb IT, van den Boomen DJ et al. MARCH6 and TRC8 facilitate the quality control of cytosolic and tail-anchored proteins. EMBO Rep. 2018;19(5).

[37] Flores-Martin J, Rena V, Angeletti S, Panzetta-Dutari GM, Genti-Raimondi S. The lipid transfer protein StarD7: structure, function, and regulation. Int J Mol Sci. 2013;14(3):6170-86.

[38] Wang YL, Faiola F, Xu M, Pan S, Human ME. ATAC Is a GCN5/PCAF-containing acetylase complex with a novel NC2-like histone fold module that interacts with the TATA-binding protein. J Biol Chem. 2008;283(49):33808-15.

[39] Seixas AI, Loureiro JR, Costa C, Ordonez-Ugalde A, Marcelino $\mathrm{H}$, Oliveira $\mathrm{CL}$ et al. A pentanucleotide ATTTC repeat insertion in the non-coding region of DAB1, mapping to SCA37, causes spinocerebellar ataxia. Am J Hum Genet. 2017;101(1):87-103.

[40] Corral-Juan M, Serrano-Munuera C, Rabano A, Cota-Gonzalez $D$, Segarra-Roca A, Ispierto $L$ et al. Clinical, genetic and neuropathological characterization of spinocerebellar ataxia type 37. Brain. 2018;141(7):1981-97.

[41] van Rootselaar AF, Aronica E, Jansen Steur EN, Rozemuller-Kwakkel JM, de Vos RA, Tijssen MA. Familial cortical tremor with epilepsy and cerebellar pathological findings. Mov Disord. 2004;19(2):213-7.

[42] Bourdain F, Apartis E, Trocello JM, Vidal JS, Masnou P, Vercueil L et al. Clinical analysis in familial cortical myoclonic tremor allows differential diagnosis with essential tremor. Mov Disord. 2006;21(5):599-608.

\section{Lorenz Peters}

Department of Neurology, University Hospital Essen, Essen, Germany

\section{Christel Depienne}

Institute of Human Genetics, University Hospital Essen, University of Duisburg-Essen, Essen, Germany

christel.depienne@uni-due.de

\section{Stephan Klebe}

Department of Neurology, University Hospital Essen, Essen, Germany

stephan.klebe@uk-essen.de 